\section{Screening for ophthalmic involvement in asymptomatic patients with metastatic breast carcinoma}

S Fenton ${ }^{1}$, EG Kemp ${ }^{1}$ and AN Harnett ${ }^{2}$
${ }^{1}$ The Ocular Oncology Unit Tennent Institute of Ophthalmology Gartnavel General Hospital Glasgow, UK

${ }^{2}$ The Beatson Oncology Centre

Western Infirmary

Glasgow, UK

Correspondence:

S Fenton

Knockion Cunningham Road

Dalkey, Dublin

Ireland

Tel: +353214922652

Fax: +353214922656

E-mail: fentons@shb.ie

\begin{abstract}
Background Breast carcinoma metastasises to the eye more frequently than is clinically recognised. The incidence is perhaps not appreciated, either because of the more common involvement and consequences of spread to major organs (such as lung, liver, or bone) or because a number of eye lesions are small and asymptomatic. Over a 6-month period, all patients with locally advanced or metastatic breast cancer were screened for ocular involvement and as a result management recommendations made. Materials and methods Between January 2001 and June 2001, 68 patients with known locally advanced or metastatic breast carcinoma were referred for a screening ophthalmic examination. The aim of the study was to assess the frequency of asymptomatic ocular metastases by breast carcinoma in visually asymptomatic patients. The recognition and early treatment of both ocular metastases and ocular manifestations of metastatic breast carcinoma are important in maximising the quality of life in this group of palliative patients. These patients were all referred and recruited from the Beatson Oncology Centre and Breast Unit at the Western Infirmary, Glasgow by the oncologist (ANH). Examination included visual acuity assessment, slit-lamp examination, tonometry, and indirect ophthalmoscopy.

Results The median time from diagnosis of breast carcinoma to ophthalmic screening was 5 years (range 6 months-23 years). No patient had any evidence of choroidal metastases on ophthalmic examination. Four patients $\mathbf{( 5 . 8 \% )}$ had ophthalmic manifestations of metastatic breast carcinoma and a further two had ocular
\end{abstract}

complications of treatment. One patient had a restrictive motility problem from a metastatic deposit to her lateral rectus muscle and another had corneal punctate epitheliopathy secondary to a seventh nerve palsy. A further patient had coarse nystagmus from cerebellar metastases and the final patient of the four had a Horner's syndrome from metastases in the neck. In addition, two patients had symptomatic dry eyes whose onset coincided with commencement of chemotherapy.

Conclusion Ophthalmic manifestations of metastatic breast carcinoma occurred in $5.8 \%$ of asymptomatic patients. Orbital metastases were documented in one patient. No case of choroidal metastases was observed in this group with advanced or metastatic disease. Therefore, patients do not need to be routinely screened particularly for choroidal metastases.

Eye (2004) 18, 38-40. doi:10.1038/sj.eye.6700535

Keywords: ocular metastases; metastatic breast carcinoma; ophthalmic screening for metastases

Introduction

Metastatic breast carcinoma is the most common primary tumour to metastasise to ocular structures. ${ }^{1}$

The incidence of ocular metastases among patients with breast carcinoma in clinical series has been reported to vary between 8 and 10\%., ${ }^{2,3}$ The occurrence of ocular metastases may not be appreciated because of the dominant clinical picture of metastases in other organs. Treatment options have increased and are prolonging survival, and thereby may increase the possibility of ocular metastases. Although the 
highly vascular posterior choroid is the most frequent site of involvement, patients can be completely asymptomatic unless vision is threatened.

The recognition of ocular manifestations of metastatic disease and early treatment of metastases are important in maximising the quality of life in these patients. We undertook a pilot study to examine the frequency of ophthalmic manifestations of metastatic breast carcinoma in visually asymptomatic patients and to determine the frequency of asymptomatic ocular metastases.

\section{Methods}

Between January 2001 and June 2001, 68 patients with known advanced or metastatic breast carcinoma were referred for a screening ophthalmic examination. These patients were visually asymptomatic. They were recruited from the Beatson Oncology Centre by the oncologist (ANH). Ethical approval was sought but felt not to be required by the Ethics Committee and so the study was explained to patients and verbal consent obtained. One patient refused to take part. Examination included visual acuity assessment, slit-lamp examination, tonometry, and indirect ophthalmoscopy.

\section{Results}

The mean age of the patients was 60 years (range 36-77, median 62 years). One of the 68 patients was a male with metastatic breast cancer. The mean time from diagnosis of breast carcinoma to ophthalmic screening was 6.3 years (median 5 years). The mean duration of metastases was 19 months. In all, $45 \%$ of patients (31) had metastases to one site, $30 \%$ of patients (21) had metastases to two sites, $9 \%$ (six) had metastases to three sites, and $11 \%$ (eight) had metastases to four sites. The remainder $5 \%$ (four) had documented widespread metastases.

The most frequent site of involvement was bone followed by lung, soft-tissue involvement, liver, CNS, and others. In total, $54 \%$ of patients had bony metastases, $30 \%$ had pulmonary metastases, $26 \%$ had soft-tissue metastases, $20 \%$ had liver metastases, and $4 \%$ had brain or CNS metastases.

At the time of ophthalmic examination, all patients were receiving systemic treatment. This included palliative radiotherapy, chemotherapy, and hormonal therapy alone or in combination. Hormonal therapy included tamoxifen, aromatase inhibitors, and medroxyprogesterone acetate.

Four patients $(5.8 \%)$ had ophthalmic manifestations of metastatic breast carcinoma. One patient had a restrictive motility problem from a metastatic deposit in the lateral rectus muscle, confirmed on CT scan and biopsied for which she received orbital radiotherapy. The onset of diplopia corresponded with her scheduled clinic visit and she was asymptomatic until this time. This same patient had a metastatic deposit to her lateral rectus muscle 2 years previously, which previously showed an excellent response to orbital radiotherapy. One patient had corneal punctate epitheliopathy secondary to lagophthalmos from a seventh nerve palsy requiring intensive lubricants and a further patient had coarse nystagmus from cerebellar metastases. Finally, one patient demonstrated a Horner's syndrome from metastases in the neck. No patient had macroscopic choroidal metastases. In addition, two patients had symptomatic dry eyes, the onset of which coincided with commencement of their chemotherapy, and were treated with topical lubricants.

Incidental eye findings causing impaired visual acuity but not subjective symptoms included posterior subcapsular lens opacities in one patient, age-related macular degeneration in one patient, uncorrected refractive error in one patient, and anisometropic amblyopia in two patients.

\section{Discussion}

The aim of our study was to determine the frequency of ocular involvement in visually asymptomatic patients with advanced or metastatic breast carcinoma and particularly to detect the presence of asymptomatic choroidal metastases where early recognition and treatment may avoid visual loss. Ophthalmic manifestations of metastatic breast carcinoma occurred in four asymptomatic patients.

Previously, much of our knowledge has come from autopsy studies. The rate of ocular metastases is expectedly higher in these studies since occult microscopic disease can be detected. Of 741 autopsy cases examined where cancer was the cause of death at John Hopkins Hospital over a 4-year period, 36 cases died of breast carcinoma. No case had macroscopic ocular metastases, but 3/36 (8.3\%) had microscopic metastases. ${ }^{4}$ Bloch and Gartner ${ }^{5}$ also observed ocular metastases in $36 \%$ of patients who died of breast cancer, and Font and Ferry ${ }^{6}$ found a similar incidence of ocular metastases in $41 \%$ of patients who died from breast cancer.

A clinical study by Mewis and Young ${ }^{3}$ found an incidence of $9 \%$ for choroidal metastases in asymptomatic patients who had metastatic breast carcinoma. In their study group, the median age was 51 years and the median interval between the diagnosis of the primary tumour and that of uveal metastases was 3 years. The median age of our patients was 11 years older and the median time to ophthalmic screening was 6 years. This is within the range when choroidal 
choroidal metastases was observed in this group with advanced metastatic disease. As a result of this study, we recommend that routine ophthalmic assessment is not performed in asymptomatic patients with advanced or metastatic breast cancer.

\section{References}

1 Bullock JD, Yanes B. Ophthalmic manifestations of metastatic breast cancer. Ophthalmology 1980; 87(10): 961-973.

2 Albert DM, Rubenstein RA, Scheie HG. Tumour metastases to the eye. Part 1 . Incidence in 213 patients with generalised malignancy. Am J Ophthalmol 1967; 63: 723-726.

3 Mewis L, Young SE. Breast carcinoma metastatic to the choroid: analysis of 67 patients. Ophthalmology 1982; 89: 147-151.

4 Eliassi-Rad B, Albert DM, Green WR. Frequency of ocular metastases in patients dying of cancer in eye bank populations. Br J Ophthalmol 1996; 80: 125-129.

5 Bloch RS, Gartner S. The incidence of ocular metastatic carcinoma. Arch Ophthalmol 1971; 85: 673-675.

6 Font RL, Ferry AP: Carcinoma metastatic to the eye and orbit: III. A clinicopathological study of 28 cases metastatic to the orbit. Cancer 1976; 38: 1326-1335.

7 Shields CL, Shields JA, Gross NE, Schwartz GP, Lally SE. Survey of 520 eyes with uveal metastases. Ophthalmology 1997; 104: 1265-1276.

8 Wiegel T, Kreusel KM, Bornfeld N, Bottke D, Stange M, Foerster MH et al. Frequency of asymptomatic choroidal metastases in patients with disseminated breast cancer: results of a prospective screening programme. $\mathrm{Br} \mathrm{J}$ Ophthalmol 1998; 82: 1159-1161.

9 Henderson JW in collaboration with Campbell RJ, Farrow GM, Garrity JA. Orbital Tumours, 3rd ed. Raven Press: New York, 1994. pp 361-375.

10 Kennedy RE. An evaluation of 820 orbital cases. Trans Am Ophthalmol Soc 1984; 82: 134-157.

11 Shields CL, Shields JA, Peggs M. Tumours metastatic to the orbit. Ophthalmic Plast Reconstruct Surg 1988; 4: 73-80.

12 Lacey B, Chang W, Rootman J. Non-thyroid causes of extraocular muscle disease. Surv Ophthalmol 1999; 44: 187-213.

13 SEER Cancer Statistics Review, 1973-1994: Tables and Graphs. National Cancer Institute: Bethesda, MA, NIH Publication No. 97-2789, 1997. 